\title{
Intravascular ultrasound online guidance during transcatheter valve replacement for native aortic stenosis or failed bioprosthesis
}

\author{
Łukasz Kalińczuk', Zbigniew Chmielak', Kamil Zieliński¹,2, Jaffar Khan³, Maciej Dąbrowski', \\ Michał Świerczewski1,2, Ilona Michałowska', Marcin Demkow ${ }^{1}$, Adam Witkowski ${ }^{1}$, Gary S. Mintz ${ }^{4}$ \\ 1 Institute of Cardiology, Warsaw, Poland \\ 2 Medical University of Warsaw, Warsaw, Poland \\ 3 MedStar Heart and Vascular Institute, MedStar Washington Hospital Center, Washington, DC, United States \\ 4 Cardiovascular Research Foundation, New York, New York, United States
}

Correspondence to: Łukasz Kalińczuk, MD, Institute of Cardiology, ul. Alpejska 42,

04-628 Warszawa, Poland phone: +483434342 , email: lukasz.kalinczuk@gmail.com Received: March 31, 2020. Revision accepted: April 16, 2020. Published online: April 23, 2020. Kardiol Pol. 2020; 78 (7-8): 762-765 doi:10.33963/KP.15307

Copyright by the Author(s), 2020
Introduction The geometry and expansion of transcatheter heart valve (THV) have an impact on paravalvular leak and subsequent leaflet deterioration, particularly after valve-in-valve procedures. ${ }^{1,2}$ Planning of transcatheter aortic valve replacement (TAVR) relies on multislice computed tomography (MSCT) along with fluoroscopy and transesophageal echocardiography. Also, a 3-dimensional reconstruction has been applied to rotational angiography to create MSCT-like images. ${ }^{3}$ The Visions PV.035 intravascular ultrasound (IVUS) catheter (Philips North America, Andover, Massachusetts, United States) with a 60-mm imaging field, is capable of tracking over 0.035- to 0.038-in guidewires used during TAVR. We used the Visions PV.035 catheter for the online assessment of the aortic root and THV geometry to provide a tomographic perspective and the highest visual resolution during TAVR for native aortic stenosis or a failed bioprosthetic aortic surgical valve.

Methods In a small case series, we enrolled patients qualified by the Heart Team upon angio-MSCT (SOMATOM, Siemens Healthineers, Erlangen, Germany) for transfemoral TAVR. The Visions PV.035 IVUS catheters were used for online guidance of the procedure.

The study was approved by the local ethics committee. Informed consent to participate in the study was obtained from all patients.

Results and discussion Patient no.1 The Heart Team qualified for transfemoral TAVR upon
angio-MSCT an 87-year-old woman with symptomatic aortic valve stenosis (mean aortic gradient, $62 \mathrm{~mm} \mathrm{Hg}$; aortic valve area, $0.5 \mathrm{~cm}^{2}$ ), normal left ventricular size and function, and the EuroSCORE II of $9.4 \%$ (baseline estimated glomerular filtration rate of $45.4 \mathrm{ml} / \mathrm{min} / 1.73 \mathrm{~m}^{2}$, diabetes mellitus, and hypertension). The Evolut R 29 mm (Medtronic, Minneapolis, Minnesota, United States) was deployed after predilation $(20 \mathrm{~mm} \times 4 \mathrm{~cm}$ Edwards Transfemoral Balloon Catheter, Edwards Lifesciences, Irvine, California, United States) (Supplementary material, Figure S1). Angiography showed aortic regurgitation and inflow outer diameter that measured $89.7 \%$ of the maximal annulus diameter ( $25.3 \mathrm{~mm}$ and $27.7 \mathrm{~mm}$, respectively) and $87.2 \%$ of the nominal inflow diameter $(25.3 \mathrm{~mm}$ and $29 \mathrm{~mm}$, respectively). The waist of the THV was overexpanded ( $25.9 \mathrm{~mm}$ versus $23 \mathrm{~mm}$ nominal size). Postdilation ( $25 \mathrm{~mm} \times 4 \mathrm{~cm}$, Edwards Lifesciences) led to an increase in inflow expansion (100.7\% of the maximal annulus diameter and $96.2 \%$ of the nominal inflow diameter) and decreased regurgitation.

A detailed analysis of the baseline aortic root by IVUS corresponded closely with MSCT, both showing an oval shape annulus with similar dimensions and no calcifications (FIGURE1, cross sections 1 and 2). Both visualized severe calcifications at the base of all 3 cusps (FIGURE 1 , cross sections 3 and 4) and no calcifications within commissural end points (FIGURE1, cross sections 6 and 7). The noncoronary cusp free margin was immobile 


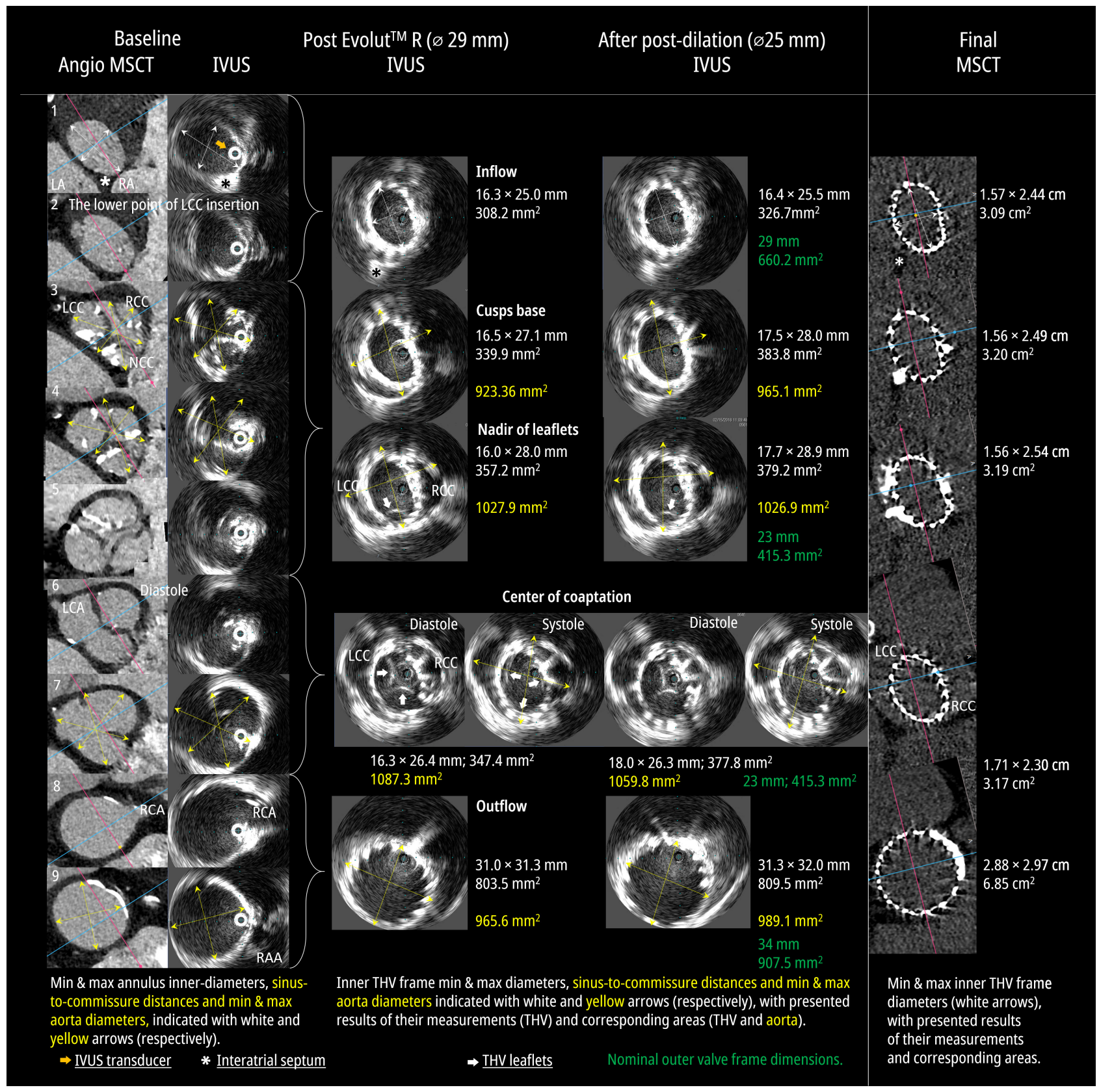

FIGURE 1 Corresponding computed tomography and intravascular ultrasound (IVUS) images of the aortic root anatomy, obtained sequentially at baseline, immediately post Evolut R $29 \mathrm{~mm}$ implantation, and after postdilation (patient no. 1). Unless marked otherwise, multislice computed tomography (MSCT) images were obtained in systole. Baseline MSCT and IVUS images correspond to: aortic annulus (cross sections 1 and 2), cusps base (cross section 3), aorta narrowest lumen cross section (cross section 4), cusps free margins (cross section 5), the level of sinuses with maximum aorta dimension (cross sections 6 and 7), and plane $45 \mathrm{~mm}$ above the annulus (cross sections 8 and 9). Procedural IVUS images correspond to: THV inflow (annulus level and cusps base), constrained segment (nadir of leaflets and center of coaptation), and outflow. Post-procedure MSCT images at levels corresponding to the procedural IVUS images. MSCT annulus measurements were $2.16 \mathrm{~cm} \times 2.82 \mathrm{~cm}, 8.1 \mathrm{~cm}$ (perimeter) and $4.84 \mathrm{~cm}^{2}$ (area). The aorta measurements at the level of the cusps were $2.94 \times 3.11 \times 3.31 \mathrm{~cm}$ (sinus-to-commissure diameters) and $7.7 \mathrm{~cm}^{2}$; at its narrowest lumen, $3.2 \times 3.33 \times 3.64 \mathrm{~cm}$ and $9.14 \mathrm{~cm}^{2}$; at the level of sinuses, $3.24 \times 3.32 \times 3.53 \mathrm{~cm}$ and $9.25 \mathrm{~cm}^{2}$; and at $45 \mathrm{~mm}$ above the annulus, $3.25 \times 3.32 \mathrm{~cm}$ and $8.49 \mathrm{~cm}^{2}$ (respectively). Corresponding baseline IVUS were as follows: at the annulus $19.3 \times 27.7 \mathrm{~mm}, 75.8 \mathrm{~mm}$ and $417.1 \mathrm{~mm}$; at the level of cusps $29.6 \times 30.6 \times 31.7 \mathrm{~mm}$ and $736.2 \mathrm{~mm}^{2}$; at its narrowest lumen cross-sectional area $31.7 \times 31.8 \times 32.6 \mathrm{~mm}$ and $910.8 \mathrm{~mm}^{2}$; at the level of the sinuses $33.6 \times 33.7 \times 35.4 \mathrm{~mm}$ and $912.8 \mathrm{~mm}^{2}$; and $45 \mathrm{~mm}$ height above the annulus plane $32.6 \times 33.3 \mathrm{~mm}$ and $822.8 \mathrm{~mm}^{2}$. Final THV inner inflow cross-sectional area was $78.3 \%$ of the baseline annulus area (with its maximal diameter ratio being $92 \%$ of the maximal baseline annulus diameter). The corresponding THV inflow area expansion was $49 \%$ of that predicted with the manufacturer's chart (with its maximal diameter ratio being $88 \%$ of that predicated). Final inner-THV frame cross-sectional areas measured at the nadir of leaflets and at the level of center of coaptation were $91 \%$ of that predicted from manufacturer's chart for both. The increase in the aortic root dimension during the TAVR procedure were $195 \mathrm{~mm}^{2}$ as assessed at the cusps base, $113 \mathrm{~mm}^{2}$ as assessed at the site of narrowest lumen, $135 \mathrm{~mm}^{2}$ as assessed at the level of sinuses, and $140 \mathrm{~mm}^{2}$ as assessed $45 \mathrm{~mm}$ above the annulus plane. Abbreviations: IVS, interventricular septum; LA, left atrium; LCA, left coronary artery; LCC, left coronary cusp; LV, left ventricle; NCC, noncoronary cusp; RA, right atrium; RAA, right atrial appendage; RCA, right coronary artery; RCC, right coronary cusp 
although not calcified and was clearly visible on IVUS (FIGURE 1 , cross sections 5 and 6). Free margins of the left and the right coronary cusps were calcified and poorly seen on IVUS due to shadowing (FIGURE 1 , cross sections 5 and 6). Whereas the right coronary take-off was easily seen with IVUS, the left coronary take-off appeared to be located too far away from the transducer (located near the commissural end point between the right coronary cusp and noncoronary cusp).

IVUS was done immediately post-Evolut $\mathrm{R}$ deployment and then after postdilation. Both runs showed an oval-shaped THV frame inflow (FIGURE1, cross sections 1 and 2), similarly to other cross sections, particularly nadir of leaflets and center of coaptation (FIGURE1, cross sections 3 and 7), whereas the outflow was circular (FIGURE 1 , cross sections 8 and 9). Evolut $R$ measured after postdilation showed that the inflow and outflow remained underexpanded as compared with baseline annulus dimensions and dimensions predicted from the manufacturer's chart. The expansion of the THV constrained segment was also smaller than nominal; however, corresponding maximal diameters (measured at the nadir of leaflets and center of coaptation) were larger than predicted ( $26 \%$ and $14 \%$ overexpansion, respectively). The mechanism of acute lumen gain post-TAVR was cusp compression (mainly the noncoronary cusp) and concomitant increase in aortic root dimensions while postdilation lead to more cusp compression, but not additional increase in the aortic root dimension. IVUS visualized normal THV leaflet motion (FIGURE1).

Patient no.2 A 91-year-old man with severe renal impairment (baseline estimated glomerular filtration rate of $33 \mathrm{ml} / \mathrm{min} / 1.73 \mathrm{~m}^{2}$ ), coronary artery disease, atherosclerosis obliterans, dyslipidemia, diabetes mellitus, and hypertension (EuroSCORE II, 39\%) presented with symptomatic significant insufficiency of the Hancock II $23 \mathrm{~mm}$ bioprosthesis (Medtronic) inserted 14 years earlier for aortic stenosis. Echocardiogram demonstrated the left ventricle with a diastolic diameter of $54 \mathrm{~mm}$, septal wall thickness of $14 \mathrm{~mm}$, and ejection fraction of 50\%. He was treated with transfemoral TAVR using Evolut R $23 \mathrm{~mm}$. Angiography showed no aortic regurgitation. THV inflow outer diameter measured $83.3 \%$ of the maximal inner-ring diameter (17.5 $\mathrm{mm}$ and $21 \mathrm{~mm}$, respectively) and $76.1 \%$ of nominal size $(17.5 \mathrm{~mm}$ and $23 \mathrm{~mm}$, respectively). The waist of the valve was underexpanded (18.6 $\mathrm{mm}$ versus $20 \mathrm{~mm}$ nominal size) (Supplementary material, Figure S2).

A detailed analysis of the baseline aortic root by IVUS corresponded closely with preprocedural MSCT (Supplementary material, Figure S3), documenting a circular bioprosthesis sewing ring with severe calcifications and similar dimensions and similar distances between the tops of stent posts (Supplementary material, Figure S3, cross section 4). Both showed similar spatial configuration of the echolucent cusps (free of calcifications or thrombus and mobile). Valve orifice dimensions on IVUS defined by the inner area in systole between the cusps in their open position were $14.6 \mathrm{~mm} \times 16 \mathrm{~mm}$ and $179.1 \mathrm{~mm}^{2}$ area (Supplementary material, Figure S3, cross section 3). The IVUS transducer was located closer to the right coronary take-off enabling good visualization, whereas the left coronary take-off appeared to be located too far away (Supplementary material, Figure S3, cross sections 4 and 5).

IVUS performed post-Evolut R implantation showed circular valve frame inflow closely adjacent to the inner surface of the bioprosthesis ring (Supplementary material, Figure S3, cross section 3 ). The other cross sections were also circular (particularly the nadir of leaflets and the center of coaptation). The inner cross-sectional area of the THV inflow was smaller than the inner ring area of the surgical valve (Supplementary material, Figure S3, cross sections 3). IVUS indicated that whereas the THV constrained segment was underexpanded at the level of the nadir of leaflets (Supplementary material, Figure S3, cross section 4), it was overexpanded at the level of the center of coaptation (Supplementary material, Figure S3, cross section 5) with an outflow dimension smaller than predicated (Supplementary material, Figure S3, cross section 6). Acute lumen gain was associated with cusp compression, but not an increase in bioprosthesis ring dimensions, with simultaneous increase in inner distances between the bioprosthesis stent posts measured at their upper points (Supplementary material, Figure S3, cross section 4). Overall aortic root dimensions (including its annulus) did not change (Supplementary material, Figure S3).

Both patients Reports of the feasibility of IVUS for TAVR did not include the analysis of THV geometry and valve-in-valve procedures. ${ }^{4,5} \mathrm{Cur}$ rently, we documented:1) concordance between quantitative and qualitative results of MSCT as compared with IVUS of a native aortic root and a failed bioprosthesis; 2) good agreement in terms of THV frame volumetric assessment; 3) mechanisms of acute lumen gain relying on cusp compression along with THV frame adaptation to the anatomy of the aortic root (particularly the geometry of cusp calcifications) with substantial increase in the aortic root dimensions or an increase in the inner distances between the bioprosthesis stent posts during the valve-in-valve procedure; and 4) the value of the online IVUS for insights into postdilation effects.

Severe calcifications are powerful reflectors of ultrasound affecting detailed real-life IVUS evaluation of patients qualified for TAVR (patient 
no. 1). IVUS visualized the annulus when there were no calcifications, calcium deposits were localized far from the transducer, deep at the base of the cusps, or were not continuous, allowing parts of the beam to penetrate. Conversely, there was substantial shadowing associated with reverberations and multiple reflections from calcium deposits at the free margins of the cusps located close to the transducer. In patient no. 2, calcium deposits occupied the valve stent (its ring and posts), but not the valve leaflets. Despite that, precise evaluation of the change in the inner-ring dimension and its relation to corresponding baseline annulus dimensions advocate IVUS use for the guidance of bioprosthetic valve fracture. Also, IVUS appears to be useful for both the BASILICA (bioprosthetic or native aortic scallop intentional laceration to prevent coronary artery obstruction) and the BI-SILICA (bicuspid scallop intentional laceration to induce circularization of the aortic implant) guidance.

Decision to postdilate (patient no. 1) was made because of substantial aortic insufficiency and space measured on angiography for more expansion of THV inflow (Supplementary material, Figure S1). Although IVUS could not document paravalvular flow directly, we visualized the site of suboptimal prosthesis expansion (annulus and constrained segment) with incomplete THV frame apposition. THV underexpansion at the upper inflow and the constrained segment (both at the nadir of leaflets and the center of coaptation) corresponded to baseline severe calcifications within the cusps' base. Maximal inner-diameters within the constrained segment were actually bigger than predicted in both patients. This is consistent with our previous volumetric quantitative MSCT analysis of supra-annular $\mathrm{THVs}^{6}$ showing that minimum outer diameter of the waist of THV was similar to the predicted diameter, but that other waist dimensions (ie, maximal diameter and area) were bigger. The above could be a consequence of inflow underexpansion which promotes waist deformation. THV dimensions increased with postdilation sized to baseline inner-annulus dimensions; however, they did not reach the nominal and were smaller than baseline inner-annulus dimensions.

MSCT for THV frame or bioprosthesis evaluation is affected by image artifacts. Angiographic measurements are made on a 2-dimensional single projection of the 3 -dimensional valve frame and require calibration. The above, along with the inconsistent $C$ arm and table settings, result in imprecise measurements of THV absolute dimensions. IVUS could be the preferred tool for the assessment of THV structural integrity and dimensions, especially among patients with renal impairment. IVUS cannot visualize coronary take-off if it is located far from the ostia; and when performing IVUS during TAVR, it is required to keep the transducer perpendicular to the aortic root or THV long axis for the most accurate measurements.

The IVUS offered online tomographic perspective with the highest visual resolution, allowing the gradual insight into the actually achieved THV dimensions and the mechanisms of therapy. Future large prospective studies should confirm our hypotheses and assess clinical utility of IVUS for TAVR guidance particularly in the setting of a heavily calcified landing zone, including balloon expandable valves and those deployed with controlled mechanical expansion as well as TAVR-in-TAVR procedures (perhaps using catheters allowing blood flow visualization).

\section{SUPPLEMENTARY MATERIAL}

Supplementary material is available at www.mp.pl/kardiologiapolska.

\section{ARTICLE INFORMATION}

ACKNOWLEDGMENTS This work was supported by a research grant funded by the Institute of Cardiology in Warsaw, Poland (2.4/VI/18; to ŁK).

CONFLICT OF INTEREST M. Dąbrowski received speaker honoraria from Boston Scientific and personal fees from Boston Scientific, Medtronic, and Edwards Lifesciences. AW is a proctor of Medtronic and Edwards Lifesciences and received speaker honoraria from Abbott, Boston Scientific, and Edwards Lifesciences. GSM has received honoraria from Boston Scientific, Philips, Medtronic, and Terumo. Other authors declare no conflict of interest with regards to this manuscript.

OPEN ACCESS This is an Open Access article distributed under the terms of the Creative Commons Attribution-NonCommercial-NoDerivatives 4.0 International License (CC BY-NC-ND 4.0), allowing third parties to download articles and share them with others, provided the original work is properly cited not changed in any way, distributed under the same license, and used for noncommercial purposes only. For commercial use, please contact the journal office at kardiologiapolska@ptkardio.pl.

HOW TO CITE Kalińczuk $Ł$, Chmielak Z, Zieliński K, et al. Intravascular ultrasound online guidance during transcatheter valve replacement for native aortic stenosis or failed bioprosthesis. Kardiol Pol. 2020; 78: 762-765. doi:10.33963/ KP.15307

\section{REFERENCES}

1 Fuchs A, De Backer O, Brooks M, et al. Subclinical leaflet thickening and stent frame geometry in self-expanding transcatheter heart valves. EuroIntervention. 2017; 13: 1067-1075.

2 Klotz S, Scharfschwerdt M, Richardt D, Sievers HH. Failed valve-in-valve transcatheter aortic valve implantation. JACC Cardiovasc Interv. 2012; 5: 591-592.

3 Rodríguez-Olivares R, El Faquir N, Rahhab Z, et al. Determinants of image quality of rotational angiography for on-line assessment of frame geometry after transcatheter aortic valve implantation. Int J Cardiovasc Imaging. 2016; 32: 1021-1029.

4 Hakim D, Ghimire G, Alli 00, et al. Large-field intravascular ultrasound for annular sizing and predicting paravalvular regurgitation during TAVR: comparisons with multidetector computed tomography and transoesophageal echocardiography. Eur Heart J Cardiovasc Imaging. 2017; 18: 1404-1413.

5 Roy DA, Bhamra-Ariza P, Asherson A, et al. First-in-man use of aortic valve ultrasound for assessment of aortic valve anatomy pre- and post-transcatheter aortic valve implantation. JACC Cardiovasc Interv. 2013; 6: 634-635.

6 Kalinczuk L, Zielinski K, Chmielak Z, et al. Abstract 15944: 384-row cardiac CT assessment of the actual transcatheter heart valve frame dimensions compared with nominal dimensions derived from manufacturers' charts. Circulation. 2018; 138: A15944. 